\title{
COMMISSION 50: PROTECTION OF EXISTING AND POTENTIAL OBSERVATORY SITES
}

\author{
(PROTECTION DES SITES D'OBSERVATOIRES EXISTANTS ET \\ POTENTIELS)
}

\author{
PRESIDENT: W. Sullivan (USA) \\ VICE-PRESIDENT: J. Cohen (UK) \\ ORGANIZING COMMITTEE: D. Crawford, B. Hidayat, S. Isobe, \\ S.-Y. Jiang, D. Malin, D. MeNally \& T. Spoelstra
}

The chief activities of the Commission for this period were the organizing of two important meetings. The first was held as Joint Discussion 5 at the Kyoto General Assembly in August 1997. The proceedings have now appeared as "Preserving the Astronomical Windows", edited by S. Isobe (1997).

\section{IAU SYMPOSIUM 196}

The second meeting was IAU Symposium 196, "Preserving the Astronomical Sky", held in Vienna in July 1999; the proceedings will be edited by W. Sullivan (USA) and J. Cohen (UK), who, with J. Andersen (Denmark, IAU), D. Crawford (USA), and H. Haubold (UN) were the chief organizers.

This meeting was notably different from the usual scientific conference. The meeting dealt with the problems of light pollution, radio interferences, and space debris that hinder astronomers' ability to study the external Universe at the exquisitely sensitive levels necessary for current research. The attendees were alarmed by the increasing "technological fog" that threatens the ability of both professional astronomers and the general public to study and appreciate the wonders of the sky. The meeting had two primary purposes: (1) sharing information on the status of the environment as it adversely affects observations, and (2) developing technical and political action plans to preserve the astronomical sky. The meeting was held at the United Nations facility in Vienna, and was also a part of UNISPACE III, a major technical and diplomatic meeting of the UN Committee for the Peaceful Uses of Outer Space. Other international sponsors were COSPAR, CIE, URSI, and the International Dark-Sky Association.

The meeting had 70 participants from 25 nations; we also employed three press officers to aid in getting out the word. After three days of talks and a marvellous banquet at the old Vienna Observatory, the fourth day was devoted to break-out sessions for brainstorming effective strategies for dealing with the issues. On the final day, these were then debated and polished further, before being approved by the entire assemblage. We worked primarily on crafting a statement that was later presented for adoption by the UNISPACE participants, with the intention that this will provide a basis for future UN discussions and perhaps eventually a treaty. IAU General Secretary J. Andersen participated in the UNISPACE meeting itself and successfully shepherded our statement such that it was indeed officially adopted in the final UN Report. This was an excellent outcome.

Our statement noted that UN space treaties have defined space as "the province of all mankind", to be protected from harmful contamination and adverse changes of all kinds, and to be utilized for the benefit of all. Further, we noted that space is already showing inexorable symptoms of over-exploitation that today affect primarily astronomy, but tomorrow will increasingly affect other users of space. We recommended that UN member states cooperate to (1) insure that frequency bands allocated to radio astronomy are indeed 
free from unwanted emissions, especially those originating in telecommunications satellites, (2) explore the concept of international radio quiet zones, regions for radio observatories protected by treaty from radio interference originating either on the ground or in space, (3) develop the concept of an "electromagnetic environmental impact statement" that would be required for all proposed space projects that might interfere with scientific research or natural, cultural, and ethical values of any nation, and (4) control light pollution for the benefit of energy conservation, the natural environment, and nighttime safety.

On the technical front, the most interesting new results for light pollution were based on quantitative calibrations newly available from the Defense Meteorological Satellite Program (DMSP). Qualitative images of the nighttime Earth showing urban areas aglow have been available for twenty years, but now one can do proper photometry based on these images. $\mathrm{S}$. Isobe (Japan) presented estimates of the money wasted annually by major cities as they send street lighting upwards: for example, US3M for LondonandUS14M for New York City. P. Cinzano (Italy) presented a marvelous new nighttime image of Europe based on DMSP data combined with a light-scattering model to show estimated sky brightness (limiting magnitude) at any given location. Back on Earth, M. Smith, Director of Cerro Tololo Inter-American Observatory, Chile, emphasized the challenges and successes he has had in dealing with local authorities to preserve his telescopes' capabilities.

Radio astronomers are making plans for the next major meeting of the International Telecommunications Union to allocate radio frequencies, in May 2000. The millimeterwavelength bands, in the past largely unallocated and little used by industry, will be a major focus for radio astronomers, especially because of all the molecular spectral lines that are vital to our understanding of the chemistry and physics of the interstellar medium. H. Butcher (The Netherlands) told of his alternative approach for seeking protection for major radio observatories, especially with the Square Kilometer Array (now in the early stages of technical development) in mind. In June 1999 the science ministers of the Organization for Economic Cooperation and Development (OECD) established a high-level task force to develop long-term solutions (such as radio quiet zones) that will safeguard humankind's radio windows on the Universe, as well as allow efficient development of commercial telecommunications. R. Ekers (Australia) and R. Fisher (USA) emphasized that radio astronomers need to be more clever in rejecting interference, using techniques that are quite feasible and known in military circles but not yet developed at observatories. Furthermore, radio (and optical) observatories have been remiss in quantitatively documenting the interference they encounter over time.

W. Flury (Germany) reported on the 100,000 objects larger than $1 \mathrm{~cm}$ now circling the Earth and menacing operations of all spacecraft, including scientific missions. While this is a serious problem, our meeting did not focus on it since it was elsewhere prominent on the UNISPACE agenda, because the major space agencies are themselves giving the problem high priority.

In summary, we achieved our goal not only to exchange information among ourselves and develop new strategies, but also to bring the issues of radio interference and light pollution to an entirely new forum, the UN. In the future, we envision a UN treaty acknowledging that the astronomical sky is the cultural heritage of all humankind, and protecting everyone's access to pristine and uncluttered skies.

W. Sullivan

President of the Commission 